\title{
Design and Verification of Instantiable Compliance Rule Graphs in Process-Aware Information Systems ${ }^{\star}$
}

\author{
Linh Thao Ly ${ }^{1}$, Stefanie Rinderle-Ma ${ }^{2}$, and Peter Dadam ${ }^{1}$ \\ 1 Institute of Databases and Information Systems \\ Ulm University, Germany \\ 2 Faculty of Computer Science \\ University of Vienna, Austria \\ \{thao.ly,peter.dadam\}@uni-ulm.de, stefanie.rinderle-ma@univie.ac.at
}

\begin{abstract}
For enterprises it has become crucial to check compliance of their business processes with certain rules such as medical guidelines or financial regulations. When automating compliance checks on process models, existing approaches have mainly addressed process-specific compliance rules so far, i.e., rules that correspond to a particular process model. However, in practice, we will rather find process-independent compliance rules that are nevertheless to be checked over process models. Thus, in this paper, we present an approach that enables the instantiation and verification of process-independent compliance rules over process models using domain models. For this, we provide an intuitive visualization of compliance rules and compliance rule instances at user level and show how rules and instances can be formalized and verified at system level. The overall approach is validated by a pattern-based comparison to existing approaches and by means of a prototypical implementation.
\end{abstract}

\section{Introduction}

In many application domains, business processes are subject to compliance rules and policies that stem from domain specific requirements such as standardization or legal regulations [1. Ensuring compliance of their business processes is crucial for enterprises nowadays, particularly since auditing and certification has become a competitive edge in many domains. Examples include certified familyfriendly enterprises being more attractive to prospective employees or clinics proving a certain standard of their audited treatments to patients. Since process models are the common way to represent business processes, business process compliance can be ensured by verifying process models to be installed in processaware information systems against imposed compliance rules. Tab. 1 summarizes quality compliance rules imposed on the software development process depicted in Fig. 1.

\footnotetext{
* This work was done within the SeaFlows project, which is funded by the German Research Foundation (DFG).
} 
Table 1. Examples of compliance rules for software development

$\mathrm{c}_{1}$ Goals have to be defined before starting the development.

$\mathrm{c}_{2}$ Each test activity has to be documented.

$\mathrm{c}_{3}$ After the development freeze, no further development activities shall take place.

$\mathrm{c}_{4}$ Before carrying out a second usability test, a necessity check after the first usability test is necessary.

$\mathrm{c}_{5}$ The testing has to be followed by an approval and the integration. Additionally, no changes shall take place between the approval and the integration.

So far, existing approaches mainly focus on checking process-specific compliance rules that correspond to certain process models [1 2 3]. One example is rule $\mathrm{c}_{1}$ (cf. Tab. 1) over process model P (cf. Fig. 1) referring to process activities define goals and start of development phase. However, in practice, compliance rules are often specified in a more general manner and not specifically in correspondence with a particular process model. Rule $\mathrm{c}_{2}$, for example, refers to a general test activity and not to a functional test as present in the software development process depicted in Fig. 1. Nevertheless, for quality assurance, it could be desired to verify $c_{2}$ over the development process. Thus, in order to support a broad spectrum of realistic compliance rules, we must enable the verification of both, process-specific and process-independent compliance rules over process models.

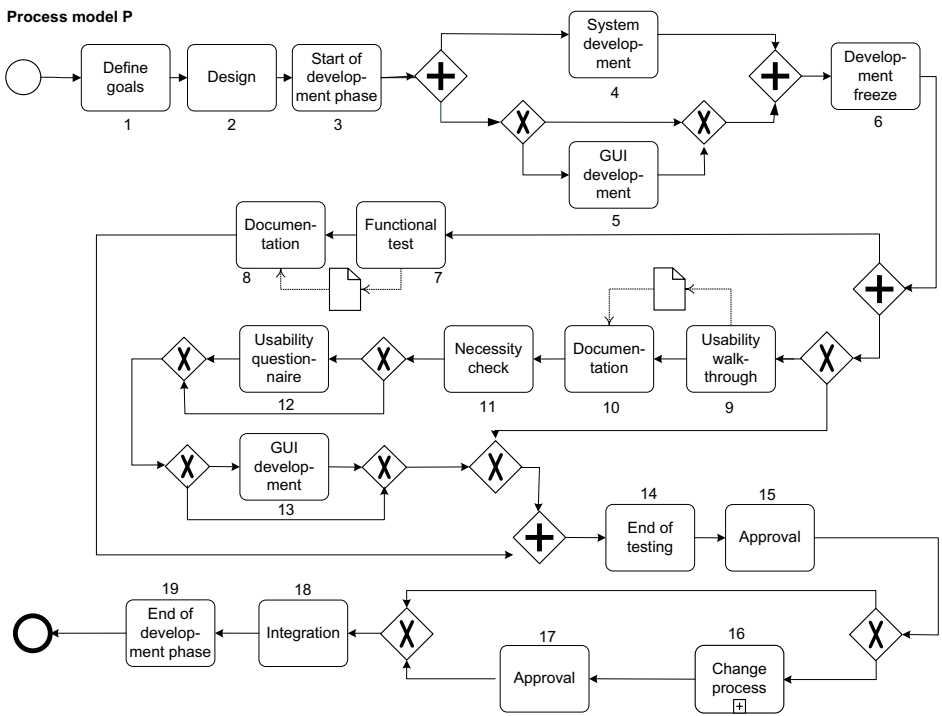

Fig. 1. Abstracted software development process (in BPMN notation) 
Connected with supporting process-independent compliance rules, it is also necessary to enable the definition of compliance rules at different design levels. At user level an intuitive, high-level representation of compliance rules is desirable. Contrary, at system level, it must be possible to conduct automatic verification of process models against compliance rules. For this, it is necessary to instantiate compliance rules over a particular process model and to equip them with formal semantics for later verification.

In this paper, we present an approach for checking process-independent compliance rules over process models. This is achieved by instantiating these compliance rules over a particular process model using a domain model. The resulting compliance rule instances can be verified separated from each other. This enables individual compliance reports as well as individual solutions in case of compliance violations. Furthermore, we show how compliance rules can be defined at different design levels. At user level, compliance rules are specified using an intuitive visualization based on graph structures. At system level compliance rule graphs are equipped with formal semantics based on First Order Logic and execution traces. The latter guarantees for independence of a particular process meta model. The overall approach is validated by a pattern-based comparison to existing approaches, by a general discussion on the co-existence of compliance rules and process models, and by means of our prototypical implementation.

Sect. 2 discusses the compliance rule instantiation approach. Sect. 3 focuses on the design of instantiable compliance rule graphs. Their counterpart formalization is presented in Sect. 4. A validation of our approach is provided in Sect. 5. We close with a related work discussion in Sect. 6] and a summary in Sect. 7

\section{Compliance Rule Instantiation}

Fig. 1 depicts the process model of a slightly abstracted software development process that we discovered within several practical student projects at Ulm University. As discussed in Sect. 1, several compliance rules might be imposed on the development process for quality and efficiency reasons (cf. Tab. 1). The basic challenge is now to check whether the process complies to these compliance rules or not. Additionally, in case of violations the system should yield helpful user feedback in precisely reporting the reason for the problem.

Compliance rules are generally defined at different abstraction levels ranging from rather abstract business policies to specific definitions [1]. To support different levels of abstraction for compliance rules, we enable the use of domain models. Based on these, compliance rules can be instantiated for certain processes. Note that domain models are demanded in many applications [4. Moreover, we may also benefit from existing ontologies such as in the healthcare domain [5]. Take for example compliance rules $c_{2}$ and $c_{4}$ (cf. Tab. 1): both refer to test activities, where $c_{2}$ is general and $c_{4}$ more specific (usability test). Fig. 2 shows an extract of the domain model belonging to the development process. A test can be more specifically modeled as a usability or functional test, where usability test can additionally distinguished into usability 

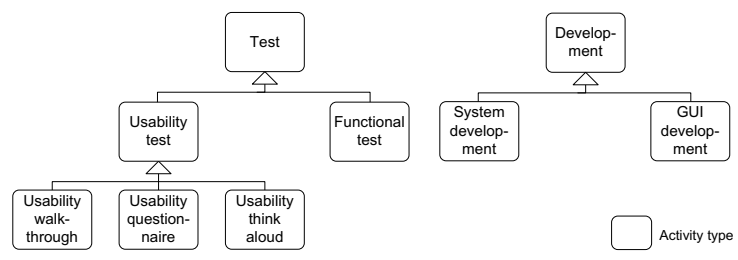

Fig. 2. An extract of the software development domain model

walkthroughs, usability questionnaires, or usability think aloud. A development might be either a system development or a GUI development.

Without any further knowledge, compliance rule $c_{2}$ cannot be evaluated over development process $\mathrm{P}$ since $\mathrm{P}$ does not contain any test activity. In fact, $\mathrm{c}_{2}$ can only be evaluated by instantiation over $\mathrm{P}$ using the corresponding domain model as depicted in Fig. 2, Based on the domain model, the general test activity referred to by $c_{2}$ can be instantiated by three more specific testing activities contained within P (i.e., usability walkthrough, usability questionnaire, and functional test). This results in three compliance rule instances $\mathrm{c}_{2_{1}}, \mathrm{c}_{2_{2}}$, and $c_{2}$ derived from $c_{2}$ (cf. Tab. 2). The usability walkthrough and the functional test are both documented according to the control flow of P. Thus, $c_{2_{1}}$ and $c_{2_{2}}$ are satisfied. However, the usability questionnaire is not documented within $\mathrm{P}$ what violates instance $\mathrm{c}_{2_{3}}$.

We see that by using instantiation the verification of $\mathrm{c}_{2}$ over $\mathrm{P}$ becomes possible. However, which benefits are offered by maintaining $\mathrm{c}_{2}$ and instantiating it "on demand" instead of replacing $\mathrm{c}_{2}$ by its instances $\mathrm{c}_{2_{1}}, \mathrm{c}_{2_{2}}$, and $\mathrm{c}_{2_{3}}$ for $\mathrm{P}$ and the corresponding domain model? The different advantages become evident when looking at the modeling, maintenance, and evolution of compliance rules on the one side and compliance checking for process models on the other side as depicted in Fig. 3 .

First of all, for more complex domain models and processes, without instantiation, the number of compliance rules might dramatically increase resulting in huge effort for compliance rule modeling and maintenance. Moreover, supporting high-level compliance rules and compliance rule instantiation eases the evolution of compliance rules. Imagine, for example, that compliance rule $c_{2}$ has to be adapted (e.g., due to changes in the quality policies) such that each test activity not only has to be documented but also has to be approved. In this case, if the strategy to explicitly model all compliance rule instances of $c_{2}$ was

Table 2. Compliance rule instances for c2, cf. Tab. 1

\begin{tabular}{l}
\hline $\mathrm{c}_{2_{1}}$ The functional test has to be documented. \\
\hline $\mathrm{c}_{2_{2}}$ The usability walkthrough has to be documented. \\
\hline $\mathrm{c}_{2_{3}}$ The usability questionnaire has to be documented.
\end{tabular}




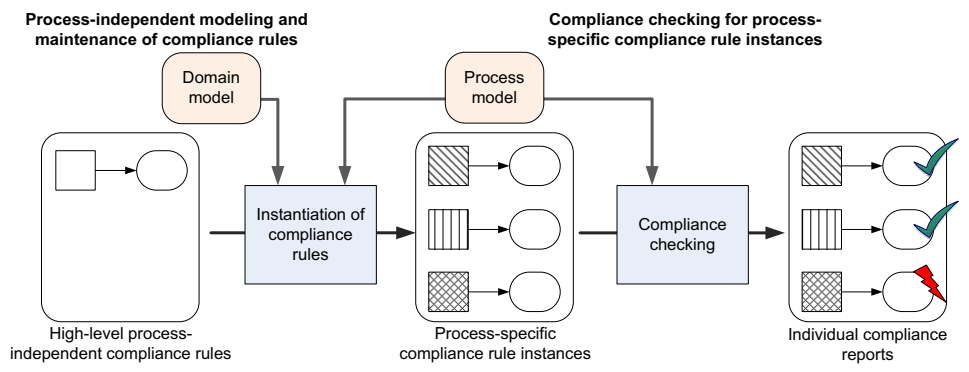

Fig. 3. The SeaFlows approach: compliance rule instantiation

applied, $\mathrm{c}_{2_{1}}, \mathrm{c}_{2_{2}}$, and $\mathrm{c}_{2_{3}}$ would have to be individually modified in order to integrate the change. By contrast, following the approach proposed in this paper, only high-level rule $\mathrm{c}_{2}$ has to be modified resulting in modified high-level rule $\mathrm{c}_{2}{ }_{2}$. c' 2 then can be reinstantiated and checked for relevant process models.

Moreover, checking compliance at instance level results in fine-granule feedback on individual violations of rule instance (cf. Fig. 3). In turn, fine-granule feedback enables fine-granule treatment of compliance rule instance violations. Let us assume, for example, that $\mathrm{c}_{2}$ is only of recommendation nature (i.e., enforcement level low). Since $c_{2_{3}}$ is violated over process model $\mathrm{P}$, the process designer might decide to completey ignore $c_{2}$. However, since $c_{2_{1}}$ and $c_{2_{2}}$ are actually fulfilled, he might prefer to ignore $c_{2_{3}}$ instead of $c_{2}$.

Altogether, using compliance rule instantiation as proposed in this paper, we achieve minimal effort for compliance rule modeling and maintenance on the one hand, but enable individual compliance checks and corresponding reports for compliance rule instances on the other hand.

\section{Instantiable Compliance Rule Graphs}

The next challenge is to design instantiable compliance rules in a way that they can be easily understood by users. This task includes representation of (highlevel) compliance rules as well as of compliance rule instances. We found that at both levels, graphs provide an intuitive visualization that can be equipped with formal semantics and verified at system level later on (cf. Sect. 4).

Process-independent Compliance Rules. To support process-independent (i.e., high-level) compliance rule graphs and as well as their instantiation over particular process models, a data model as depicted in Fig. 4 is needed: a process model consists of a set of nodes distinguished by their node id to which activities are assigned. An activity is assigned to activity types. An activity type may be the sub-type of another activity. For example, the activity type usability walkthrough is a sub-type of the activity type usability test (cf. domain model in Fig. 2). 


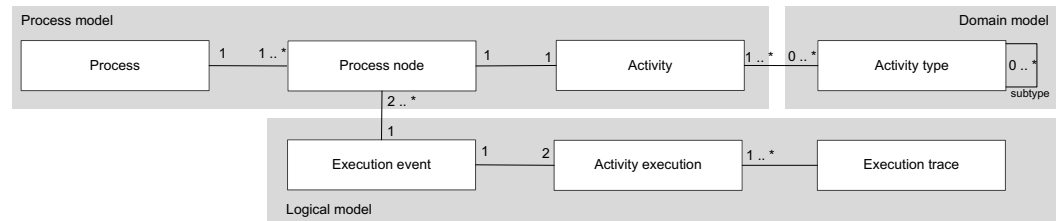

Fig. 4. The data model for instantiable compliance rules

When looking at compliance rules $c_{1}$ to $c_{5}$ (cf. Tab. 1), it can be observed that compliance rules mostly reflect certain patterns. Typically, compliance rules require the occurrence and/or the absence of certain activities (occurrence/absence patterns). For $c_{1}$, for example, activity define goals should occur before starting the development. By contrast, for $\mathrm{c}_{3}$ further development activities should be absent if activity development freeze has taken place. Furthermore, it can be observed that the occurrence or absence of certain activities is often conditional upon the occurrence or absence of other activities. Thus a compliance rule mostly comprises a triggering part (denoted as antecedent pattern) and a consequence pattern. To be able to instantiate process-inpdedendent compliance rule graphs later on, each node of a rule graph is associated with an activity type from the domain model (cf. Fig. 4). Directed edges connecting the nodes represent predecessor relations.

Based on these observations compliance rules $c_{1}$ to $c_{5}$ can be described by compliance rule graphs as depicted in Fig. 5. Compliance rule graph $\mathrm{c}_{1}$, for example, states that the execution of activity start of development process has to be directly or indirectly preceded by the execution of the activity define goals. Compliance rule graph $c_{5}$ states that the execution of activity end of testing has to be succeeded by an execution of approval and integration. Between these two executions, however, no execution of the change process must occur. Note that compliance rule graphs might also contain data information as data object Test for $\mathrm{c}_{2}$. How to integrate and evaluate such data information into compliance rules is subject to our future research.

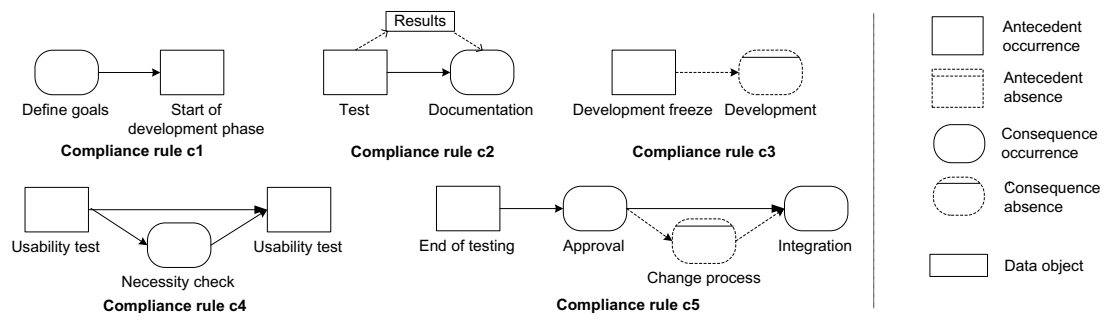

Fig. 5. Process-independent compliance rule graphs 
Process-specific Instantiation of Compliance Rules . Generally, it must be possible to define compliance rule graphs independent of a particular process model. For later verification, however, it is necessary to instantiate independent compliance rule graphs over process models.

The instantiation of compliance rule graphs is accomplished by "binding" antecedent compliance rule nodes to nodes of the process model (reflected by their node ids) with the associated activity type. Instantiation of compliance rule $c_{2}$ (cf. Fig. 5) over process model P (cf. Fig. 1) using the domain model as depicted in Fig. 2, for example, results in three compliance rule instances as depicted in Fig. 6. Visualizing compliance rules that way, the user is able to locate exactly, which occurrences of a compliance rule are relevant, which are satisfied, and which are violated.

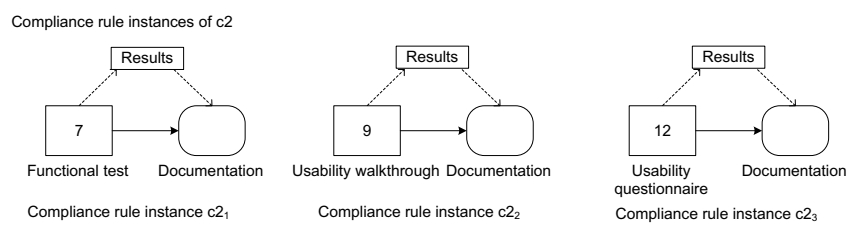

Fig. 6. Process-specific compliance rule instances

\section{Formalization and Verification of Compliance Rules}

The graphical compliance rule notation provides for more intuitive modeling of compliance rules since it hides formal details from the user. However, as we will show in this section, each compliance rule graph corresponds to a logical formula with defined semantics. The latter is necessary to enable the verification of processes against imposed compliance rules.

\subsection{On Formalizing Compliance Rules}

We opted for first-order predicate logic (FOL) to formalize compliance rules for several reasons. The use of a general and expressive logic such as FOL enables the extension of our approach in order to support further types of compliance rules not supported so far (e.g., authorization or organizational model compliance rules). Moreover, FOL allows for the definition of the antecedentconsequence-structure of compliance rules in a straight-forward and elegant manner. The nodes of compliance rule graphs are mapped to variables while properties of compliance rule graph nodes and the relations between nodes are mapped to corresponding predicates in a FOL formula. Due to lack of space we abstain from a complete definition here, but rather informally describe the structure in the following. The general structure of a compliance rule is as follows. 
Structure 1 (Compliance rule). Let $A_{\mathcal{T}}$ be the set of activity types of the domain. Then, a compliance rule is of the following form:

[true | antecedentOccurrencePatterns | antecedentAbsencePatterns | antecedentOccurrencePatterns $\wedge$ antecedentAbsencePatterns]

$\rightarrow$

consequence $\vee$ consequence $\vee \cdots \vee$ consequence

consequence $:=$

[consequenceOccurrencePatterns | consequenceAbsencePatterns |

consequenceOccurrencePatterns $\wedge$ consequenceAbsencePatterns]

In Struct. 1 the antecedent is either empty (i.e., the compliance rule is always activated) or consists of an antecedent pattern. The latter can be composed from occurrence patterns defining the occurrences of activity executions that activate the compliance rule. Compliance rule $\mathrm{c}_{2}$ (cf. Fig. (5), for example, is activated by the occurrence of an activity execution associated to the activity type test while compliance rule $c_{4}$ is activated by a more complex occurrence pattern (namely the sequence of two activity executions). The antecedent pattern may also consist of absence patterns defining the absence of particular activity executions. This allows for refining the occurrence pattern by putting additional conditions on the absence of activity executions (for example, to express patterns such as "if no approval takes place between the end of development and the integration"). If the antecedent of a compliance rule applies, one of the rule's consequence patterns must also apply in order to satisfy the rule. Each consequence pattern, in turn, may consists of occurrence as well as absence patterns and corresponding relations. Compliance rule $c_{3}$ (cf. Fig. 5), for example, has a consequence absence pattern in its consequence part while the consequence part of compliance rule c5 is composed from both consequence occurrence and consequence absence patterns.

The formula for compliance rule $c_{2}$ is given below. It expresses that each activity execution associated to the activity type test has to be followed by an activity execution associated to the activity type documentation with the same results data object. This is a process-independent compliance rule, since it only references to activity types from the domain model (cf. Fig. 22).

\section{Compliance rule c2}

$\forall t($ ActivityType $(t$, test $) \rightarrow \exists d:($ ActivityType $(d$, documentation $) \wedge \operatorname{Pred}(t, d) \wedge$ results $(t)=$ results $(d)))$

Based on the development process (cf. Fig. 1), we can identify the process nodes that are associated to the activity types referenced in the formula (namely test and documentation). The formula for $\mathrm{c}_{2}$ can be adapted accordingly by refering to activity executions associated to particular nodes in the process.

\section{Process-specific compliance rule $\mathbf{c}_{2}$}

$\forall t($ ProcessNode $(t, 7) \vee$ ProcessNode $(t, 9) \vee$ ProcessNode $(t, 12) \rightarrow$

$\exists d:(\operatorname{ActivityType}(d$, documentation $) \wedge \operatorname{Pred}(t, d) \wedge$ results $(t)=$ results $(d)))$ 
Based on this, we obtain the resulting compliance rule instances of c2 as follows.

$\mathbf{c}_{2_{1}}: \operatorname{ProcessNode}(t, 7) \rightarrow \exists d:($ ActivityType $(d$, documentation $) \wedge \operatorname{Pred}(t, d) \wedge$ results $(t)=$ results $(d))$

$\mathbf{c}_{2_{2}}: \operatorname{ProcessNode}(t, 9) \rightarrow \exists d:($ ActivityType $(d$, documentation $) \wedge \operatorname{Pred}(t, d) \wedge$ results $(t)=$ results $(d))$

$\mathbf{c}_{2_{3}}: \operatorname{ProcessNode}(t, 12) \rightarrow \exists d:($ ActivityType $(d$, documentation $) \wedge \operatorname{Pred}(t, d) \wedge$ results $(t)=$ results $(d))$

\subsection{Interpretation of Compliance Rules}

So far we showed that compliance rules are represented by FOL formulas. To round up the formalization of compliance rules, we also have to provide formal semantics for these formulas. The formal semantics has to be defined over an adequate logical model, that serves as interface between the compliance rule perspective and the process perspective. To support a variety of business process models, the logical model must be independent of a particular process metamodel. As discussed in our previous work in 6 637] execution traces are a suitable logical model since they are applicable to any process meta-model with formal execution semantics.

Generally, depending on their particular purpose, execution traces comprise different kinds of information. At minimum, execution traces store information on the execution of activities for a particular process instance (e.g., start, end, or start/end events for activity executions). Additional information might comprise actor assignments, input or output data, and timestamps (see, for example, the MXML execution traces used in ProM [8]). In the context of this paper, it is important to be able to instantiate compliance rules over process models. Thus, within execution traces, it should be possible to distinguish between concepts such as nodes and activities (cf. data model in Fig. 4). Thus, we define an ordered execution trace $\sigma$ over a process model $\mathrm{P}$ as follows:

$\sigma_{P}:=\left\langle e_{1}, \ldots, e_{k}>\right.$ with

$e_{i} \in\{$ Start(activity, node, timestamp), End(activity, node, timestamp)\} where

- activity denotes the activity event $e_{i}$ is associated with

- node denotes the process node an activity is associated with

- timestamp represents an abstract timestamp

For our formal interpretation of compliance rules, we need the activity-oriented view $\sigma_{P}^{\prime}$ of event-based execution traces $\sigma_{P} . \sigma_{P}^{\prime}$ represents the ordered activity executions in $\sigma_{P}$ :

$\sigma_{P}^{\prime}:=<a_{1}, \ldots, a_{m}>$ with

$a_{x}=\left(\right.$ activity $_{x}$, node $_{x}$, startTime $_{x}$, endTime $\left._{x}\right), x=1, . ., m$ with $\exists e_{i}, e_{j} \in \sigma_{P}$ :

$-e_{i}=\operatorname{Start}\left(\right.$ activity $_{x}$, node $_{x}$, startTime $\left._{x}\right)$,

$-e_{j}=\operatorname{End}\left(\right.$ activity $_{x}$, node $_{x}$, endTime $\left._{x}\right)$, 
$-i<j$ and $\nexists e_{l} \in \sigma_{P}: i<l<j$ and $e_{l}=\operatorname{End}\left(\right.$ activity $_{x}$, node $\left._{x}, \ldots\right)$ and

$-\forall a_{r}, a_{p}: r<p \Rightarrow$ startTime $_{a_{r}}<$ startTime $_{a_{p}}$

Based on the notion of execution traces and activity executions we can provide a default interpretation of compliance rules as follows. The interpretation relates the predicates in the compliance rule formula to activity executions in the execution trace. In the following, we focus on correct execution traces (e.g., each start event has a corresponding end event).

Definition 1 (Interpretation of compliance rules). Let $A_{\mathcal{T}}$ be the set of activity types of the domain model. Let $\sigma^{\prime}=<a_{1}, \ldots, a_{m}>$ be an activity-oriented view of an execution trace. Then, the interpretation over $\sigma^{\prime}$ over $A_{\mathcal{T}}$ is a tuple $I_{\sigma^{\prime}}=<D_{\sigma^{\prime}}, d_{\sigma^{\prime}}>$ with:

$D_{\sigma^{\prime}}$ is the domain of the interpretation $I_{\sigma^{\prime}}$ with $D_{\sigma^{\prime}}:=\left\{a_{1}, \ldots, a_{m}\right\}$

Let further $N_{\sigma^{\prime}}=\left\{n \mid \exists a=\right.$ (activity $_{a}$, node $_{a}$, startTime $_{a}$, endTime end $\left._{a}\right) \in D_{\sigma^{\prime}}$ : $n=$ node $\left._{a}\right\}$ be the set of process nodes associated to activity executions in $\sigma^{\prime}$. $d_{\sigma^{\prime}}$ is a function interpreting the predicates ActivityType, ProcessNode, and Pred 11 over $\sigma^{\prime}$ as follows:

$-d_{\sigma^{\prime}}($ ActivityType $) \mapsto\left\{(a, A), a=\left(\right.\right.$ activity $_{a}$, node $_{a}$, startTime $_{a}$, endTime $\left._{a}\right)$ $\in D_{\sigma^{\prime}}, A \in A_{\mathcal{T}} \mid$ activity $_{a}=A \vee$ activity $_{a}$ is a subtype of $\left.A\right\}$

$-d_{\sigma^{\prime}}($ Process Node $) \mapsto\left\{(a, n), a=\left(\right.\right.$ activity $_{a}$, node $_{a}$, startTime $_{a}$, endTime $\left._{a}\right)$ $\in D_{\sigma^{\prime}}, n \in N_{\sigma^{\prime}} \mid n=$ node $\left._{a}\right\}$

$-d_{\sigma^{\prime}}($ Pred $) \mapsto\left\{(a, b), \quad a=\right.$ (activity $_{a}$, node $_{a}$, startTime $_{a}$, endTime $\left._{a}\right)$ $\in D_{\sigma^{\prime}}, b=\left(\right.$ activity $_{b}$, node $_{b}$, startTime $_{b}$, endTime $\left._{b}\right) \in D_{\sigma^{\prime}} \mid$ endTime $_{a}<$ startTime $\left._{b}\right\}$

Based on Def. 1 compliance rule formulas can be interpreted over execution traces. Def. 2 provides the formal criteria necessary for compliance verification.

Definition 2 (Satisfaction of compliance rules). Let $P$ be a process model and let $\Sigma_{P}^{\prime}$ be the set of all activity-oriented views on execution traces of $P$ (i.e., all traces $P$ is able to produce). Let further $\sigma_{P}^{\prime} \in \Sigma_{P}^{\prime}$ be one activity-oriented view of an execution trace of $P$.

We say $\sigma_{P}^{\prime}$ satisfies $c$ (notation: $\sigma_{P}^{\prime} \models c$ ) if and only if:

$I_{\sigma_{P}^{\prime}}=c$.

We say $P$ satisfies $c$ (notation: $P \models c$ ) if and only if:

$\forall \sigma_{P}^{\prime} \in \Sigma_{P}^{\prime}$ holds $\sigma_{P}^{\prime} \models c$.

To illustrate the formal semantics defined above, consider again compliance rule $c_{5}$ (cf. Fig. 5) and the development process (cf. Fig. 11). Based on the execution traces that the development process can produce, Def. 1 can be applied to verify the development process against $c_{5}$. Informally, the straight-forward way to verify compliance rules corresponds to reachability analysis as applied for checking the soundness of process models [9]. In all execution traces of the process, there is an

${ }^{1}$ Constants in a compliance rule formula (i.e., node identifiers in compliance rule instances) are mapped to themself and hence, are ommitted in the interpretation. 
occurrence of end of testing (node 14). Hence, compliance rule $c_{5}$ is activated in all executions of the development process. After end of testing, either the upper (case 1) or the lower split branch (case 2) is executed. In the first case, the corresponding execution trace contains the execution of the approval (node 15), directly followed by the execution of the integration (node 18). Hence, this trace satisfies $c_{5}$. In the second case, the change process (node 16) is executed after the approval (node 15). However, another approval activity (node 17) is executed afterwards, that is directly followed by the integration. According to the interpretation from Def. 1, $c_{5}$ is also satisfied in the second case.

\section{Discussion and Validation}

Generally, different scenarios of integrating business processes and compliance rules are conceivable. At the one side of the spectrum, all relevant compliance rules might be integrated (as far as possible) within the process model. This might be achieved by a multitude of partly nested alternative branchings. Full merging of rules into process models could be desirable for scenarios with simple process models and compliance rules that are mandatory for all process models. However, this approach has also several drawbacks. The first one is the possibly enormous complexity of the resulting process models in case of a multitude of compliance rules imposed on them. This can be compared to approaches for process configuration and variants, keeping all variants or rules within one model. As research has discussed, for many scenarios, this approach quickly leads to overly complex process models that cannot be understood by users any longer [10. In addition, from our practical examples, we know that often not all compliance rules are mandatory, but rather have a suggesting character. Examples include medical guidelines that might be overruled by the doctor at any time. However, if all compliance rules are "hard-wired" within the process models, this optional character gets lost.

On the other side of the spectrum, we could also think of representing process models and compliance rules entirely as rules, for example within rule engines such as ILOG JRules or by using declarative approaches such as ConDec [1]. Aside from the fact that declarative process description can be covered by our approach, it cannot be neglected that in practice, most process models are described and automated using graph-based notations. Specifically, in practice, we will often find a coexistence of business process (models) and compliance rules imposed on them. Another reason for this coexistence is that compliance rules might be introduced after the process model has been designed and enacted.

For these reasons, we mainly aim at supporting the coexistence of process models and compliance rules which complies to approaches that aim at balancing flexibility and control by combining imperative and declarative process description [12. However, since our logical model is based on execution traces (cf. Sect. 31), SeaFlows can also deal with pure process-model-based and declarative scenarios. In the latter case, execution traces are a suitable representation of process instances defined by following the imposed constraints 13 . 


\subsection{Pattern-Based Validation}

We collected recurring compliance rule patterns from literature and modeled them using the SeaFlows compliance rule formalism. Many pattern-based approaches [141513] base their patterns on patterns collected by Dwyer and Corbett [16]. Simple (particularly binary) patterns, such as global scope presence, global scope absence, after scope absence, before scope absence, response, and precedence can be modeled similarly to some of the compliance rules from Fig. 5 (e.g., compliance rule c3 corresponds to after scope absence). Hence, we omit the illustration of these patterns due to space limitations. Three advanced patterns [14 16] are depicted in Fig. 7. The response with absence rule states that A must be followed by an occurrence of $\mathrm{C}$ without $\mathrm{B}$ occurring in the meantime. The precedence with absence rule states that $\mathrm{C}$ must be preceeded by A such that no B occurs in between. The after scope precedence chain states that the sequence A... B must occur between S and a succeeding occurrence of C.

In constrast to approaches based on a limited set of patterns and respective combinations thereof using the logical conjunction (cf. Sect. 6), the SeaFlows approach is compositional. It allows for modeling compliance rules with an
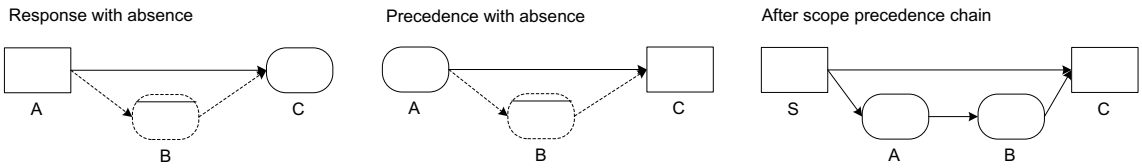

Fig. 7. Advanced compliance rule patterns 14

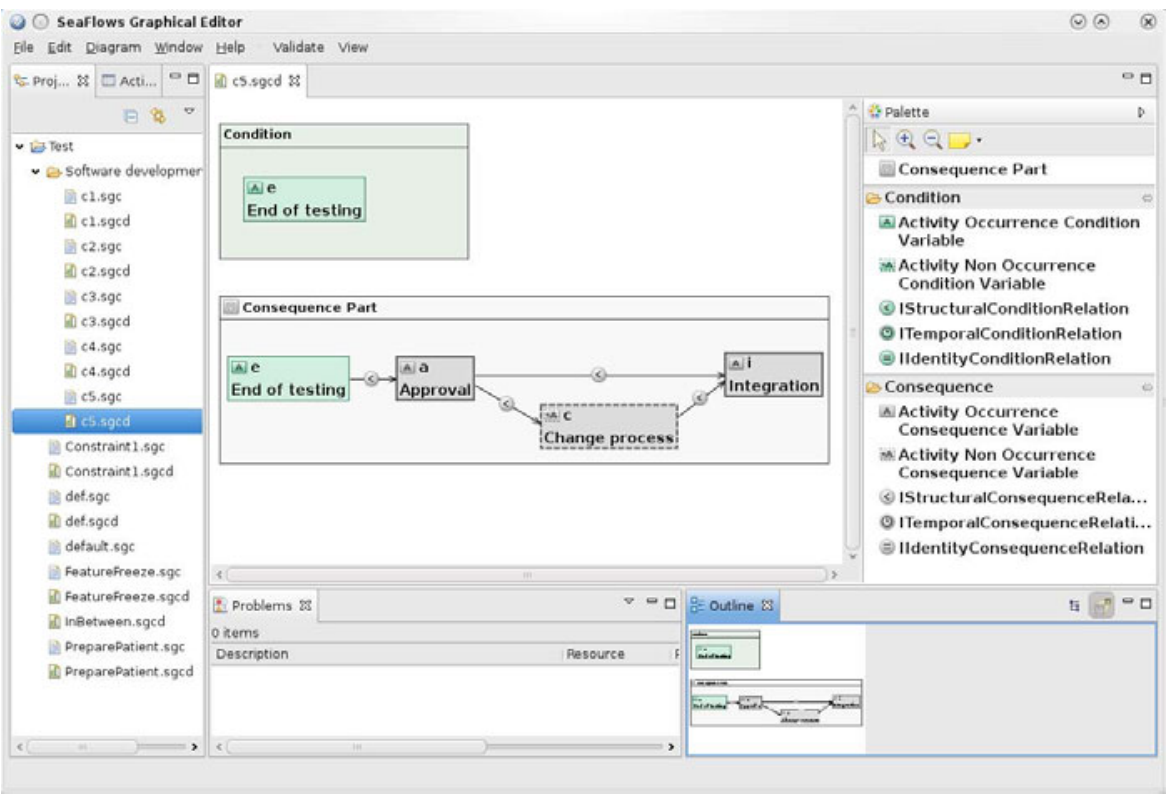

Fig. 8. The SeaFlows graphical compliance rule editor 
antecedent and a consequence part. The modeled compliance rule graphs can be automatically mapped to logical formulas (cf. Sect. 4).

\subsection{Technical Validation}

Fig. 8 shows the SeaFlows compliance rule editor. It allows for separately modeling the antecedent and consequence patterns of compliance rules (depicted in separate boxes). The SeaFlows editor is integrated into the AristaFlow BPM Suite that is based on ADEPT [6]. Each compliance rule node can be assigned an activity type from the AristaFlow Activity Repository. The latter also provides the activity types used to model processes and serves as domain model in our implementation. For convenient compliance rule modeling, the SeaFlows editor allows for modeling parametrized recurring compliance rules patterns. If the user wants to model a compliance rule with the same structure, he may apply the parametrized compliance rule pattern.

\section{Related Work}

Many approaches have been proposed in literature to model compliance rules. We focus on approaches for modeling compliance rules over the occurrence and ordering relations of activity executions within a process with the goal of process verification. Existing approaches range from rather informal annotations of process models with compliance rules, over formal languages, to visual patterns.

To enable the verification of processes against imposed compliance rules, the latter have to provide formal semantics. This requirement is met by compliance rules specified in formal languages. Among the formal languages proposed for compliance rule modeling, we often come across temporal logics such as linear temporal logic (LTL) or computational tree logic (CTL) [17. Both are fragments of first-order logic (FOL). Due to its linear time semantics, which is more suitable in the business process context [18, LTL has been clearly prefered over CTL which has branching time semantics. Albeit its expressiveness to model compliance rules with regard to the occurrence and ordering relations of activities, pure LTL has limitations when it comes to the incorporation of context conditions within compliance rules. This is due to the fact that one cannot directly address a particular state in LTL. Hence, to ensure the extendability of our approach, we opted for the formalization in FOL. The formal contract language (FCL), designed to specify business contracts, can also be applied to model compliance requirements 1920. However, FCL is based on a rather state-oriented than activity-oriented paradigm. In general, it requires certain skills to model compliance rules using a formal language which might be an obstacle to the practical application of corresponding approaches.

Due to the difficulties of modeling compliance rules using formal languages in practice, many approaches from literature suggest visual notations to hide the formal details from the modeler. In [18, Liu et al. propose a graphical business property specification language (BPSL) that is based on linear temporal logic. 
BPSL provides visual notations for logical operators. In addition, it defines dedicated operators for recurring logical patterns. In contrast to our approach, BPSL does not support the explicit structure of antecedent and consequence patterns within a compliance rule.

Other approaches aim at establishing a set of recurring compliance rule patterns. The patterns are either given visually [211411] or are organized in some kind of a pattern ontology 15/2212. Generally, these patterns are based on property patterns collected by Dwyer and Corbett [16]. Each pattern, in turn, can be mapped to a logical formula (e.g., in LTL). This enables the formal verification. Clearly, establishing set of rule patterns recurring in a business domain is an effective approach to facilitate compliance rule modeling. This can also be accomplished with our compliance rule language. Although the rule patterns can usually be combined using the logical conjunction, a fixed set of patterns can still be too restrictive for particular application scenarios. In these case, compositional approaches such as our approach are advantageous.

\section{Summary and Outlook}

We presented an approach to support the design and verification of compliance rules at different abstraction levels ranging from high-level, process independent rules to rules specified over particular process models. The main challenge was to enable the verification of process models against process-independent compliance rules. We solved this by introducing the mechanism of compliance rule instantiation using domain models and showed that instantiation results in many advantages such as easening design, mainenance, and evolution of compliance rules as well as fine-granule compliance reports. Moreover, we introduced an intuitive visualization of compliance rules and instances together with their formal semantics based on FOL and execution traces. Finally, we showed the feasability of our approach based on a pattern-based validation and by means of our powerful prototype. In future work, we will equip compliance rule graphs with operational semantics to enable more efficient compliance checking. In addition, we will further investigate on runtime issues. At runtime when process instances are created from process models and executed, the compliance with imposed compliance rules may change [7]. That is why it can become necessary to monitor the compliance during process execution.

\section{References}

1. Sadiq, S., Governatori, G., Naimiri, K.: Modeling control objectives for business process compliance. In: Alonso, G., Dadam, P., Rosemann, M. (eds.) BPM 2007. LNCS, vol. 4714, pp. 149-164. Springer, Heidelberg (2007)

2. Awad, A., Decker, G., Weske, M.: Efficient compliance checking using BPMN-Q and temporal logic. In: Dumas, M., Reichert, M., Shan, M.-C. (eds.) BPM 2008. LNCS, vol. 5240, pp. 326-341. Springer, Heidelberg (2008)

3. Ly, L.T., Rinderle-Ma, S., Dadam, P.: Integration and verification of semantic constraints in adaptive process management systems. Data and Knowledge Engineering 64, 3-23 (2008) 
4. Filipowska, A., Hepp, M., Kaczmarek, M., Markovic, I.: Organisational ontology framework for semantic business process management. In: BIS 2009. LNBIP, vol. 21, pp. 1-12. Springer, Heidelberg (2009)

5. Kumar, A., Smith, B., Pisanelli, D., Gangemi, A., Stefanelli, M.: An ontological framework for the implementation of clinical guidelines in health care organizations. Stud. Health Technol. Inform. 102, 95-107 (2004)

6. Rinderle, S., Reichert, M., Dadam, P.: Flexible support of team processes by adaptive workflow systems. Distributed and Parallel Databases 16, 91-116 (2004)

7. Ly, L.T., Rinderle-Ma, S., Göser, K., Dadam, P.: On enabling integrated process compliance with semantic constraints in process management systems. Information Systems Frontiers (2009) (accepted for publication)

8. van der Aalst, W., et al.: Prom 4.0: Comprehensive support for real process analysis. In: Kleijn, J., Yakovlev, A. (eds.) ICATPN 2007. LNCS, vol. 4546, pp. 484-494. Springer, Heidelberg (2007)

9. van der Aalst, W.: Verification of workflow nets. In: Int'l Conf. on Application and Theory of Petri Nets, pp. 407-426 (1997)

10. Hallerbach, A., Bauer, T., Reichert, M.: Managing process variants in the process lifecycle. In: Proc. ICEIS 2008, pp. 154-161 (2008)

11. Pesic, M., Schonenberg, M., Sidorova, N., van der Aalst, W.: Constraint-based workflow models: Change made easy. In: Meersman, R., Tari, Z. (eds.) OTM 2007, Part I. LNCS, vol. 4803, pp. 77-94. Springer, Heidelberg (2007)

12. Sadiq, S., Orlowska, M., Sadiq, W.: Specification and validation of process constraints for flexible workflows. Inf. Syst. 30, 349-378 (2005)

13. Pesic, M.: Constraint-Based Workflow Management Systems: Shifting Control to Users. PhD thesis, Eindhoven University of Technology (2008)

14. Awad, A., Weske, M.: Visualization of compliance violation in business process models. In: Proc. BPI 2009 (2009)

15. Yu, J., Manh, T.P., Hand, J., Jin, Y.: Pattern-based property specification and verification for service composition. CeCSES Report SUT. CeCSES-TR010, Swinburne University of Technology (2006)

16. Dwyer, M.B., Avrunin, G.S., Corbett, J.C.: Patterns in property specifications for finite-state verification. In: Proc. ICSE 1999, pp. 411-420 (1999)

17. Ghose, A., Koliadis, G.: Auditing business process compliance. In: Krämer, B.J., Lin, K.-J., Narasimhan, P. (eds.) ICSOC 2007. LNCS, vol. 4749, pp. 169-180. Springer, Heidelberg (2007)

18. Liu, Y., Müller, S., Xu, K.: A static compliance-checking framework for business process models. IBM Systems Journal 46, 335-361 (2007)

19. Governatori, G., Hoffmann, J., Sadiq, S., Weber, I.: Detecting regulatory compliance for business process models through semantic annotations. In: Proc. BPD $2008(2008)$

20. Lu, R., Sadiq, S., Governatori, G.: Compliance aware process design. In: ter Hofstede, A.H.M., Benatallah, B., Paik, H.-Y. (eds.) BPM Workshops 2007. LNCS, vol. 4928, pp. 120-131. Springer, Heidelberg (2008)

21. van der Aalst, W., Pesic, M.: DecSerFlow: Towards a truly declarative service flow language. In: Bravetti, M., Núñez, M., Zavattaro, G. (eds.) WS-FM 2006. LNCS, vol. 4184, pp. 1-23. Springer, Heidelberg (2006)

22. Namiri, K., Stojanovic, N.: Pattern-based design and validation of business process compliance. In: Meersman, R., Tari, Z. (eds.) OTM 2007, Part I. LNCS, vol. 4803, pp. 59-76. Springer, Heidelberg (2007) 